Article

\title{
Equiprobability, Entropy, Gamma Distributions and Other Geometrical Questions in Multi-Agent Systems
}

\author{
Ricardo López-Ruiz $^{1,3, \star}$, Jaime Sañudo ${ }^{2,3}$ and Xavier Calbet ${ }^{3}$ \\ ${ }^{1}$ Department of Computer Science, Universidad de Zaragoza, Pedro Cerbuna 12, Zaragoza 50009, \\ Spain \\ ${ }^{2}$ Department of Physics, Universidad de Extremadura, Avda. de Elvas s/n, Badajoz 06071, Spain; \\ E-Mail: jsr@unex.es (J.S.) \\ ${ }^{3}$ BIFI, Universidad de Zaragoza, Corona de Aragón 42, Zaragoza 50009, Spain; \\ E-Mail: xcalbet@googlemail.es (X.C.) \\ * Author to whom correspondence should be addressed; E-Mail: rilopez@unizar.es; \\ Tel.: +34-976-761-134; Fax: +34-976-761-132.
}

Received: 3 November 2009 / Accepted: 30 November 2009 / Published: 2 December 2009

\begin{abstract}
A set of many identical interacting agents obeying a global additive constraint is considered. Under the hypothesis of equiprobability in the high-dimensional volume delimited in phase space by the constraint, the statistical behavior of a generic agent over the ensemble is worked out. The asymptotic distribution of that statistical behavior is derived from geometrical arguments. This distribution is related with the Gamma distributions found in several multi-agent economy models. The parallelism with all these systems is established. Also, as a collateral result, a formula for the volume of high-dimensional symmetrical bodies is proposed.
\end{abstract}

Keywords: multi-agent systems; equiprobability; economic models; Gamma distributions

Classification: PACS 87.23.Ge, 05.90.+m, 89.90.+n 


\section{Introduction}

Nowadays different approaches to study economic systems can be taken within a multi-agent framework. Econophysics applies the techniques and tools of statistical physics to the understanding of this type of systems [1]. From this point of view, an economic system is regarded as a set of many agents exchanging money or trading with products of the most diverse origins. This exchange, which can be done in a deterministic or in a random way, finally generates an asymptotic wealth distribution. One of the goals of econophysics is to propose different models for the agents' interactions and, by making the respective simulation experiments, to try to reproduce in a statistical way the real asymptotic wealth distribution in society [2].

In this paper, a general multi-agent system evolving under an additive constraint is considered. The geometrical properties of this system in phase space are exploited in order to obtain its statistical behavior. A striking coincidence is observed. Some dynamical mechanisms that have been proposed in the literature to model the interaction among agents in economic systems [3-9] provoke the same statistical results in the asymptotic wealth distribution than those derived from the geometrical properties in our multi-agent system under the assumption of equiprobability. This fact bring us to speculate on the possible close relationship that there exists between the local interactions among the agents in the former economic systems and the global geometrical conformation in phase space of our general system. Hence, with an adequate change of coordinates, we suggest that those economic systems asymptotically evolve in an equiprobable way over the volume of accessible states in the transformed phase space.

We start in Section 2 by recalling the derivation done for some particular geometries for the cases in which the constraint has a linear or quadratic dependence on the variables defining the agents. Then, in Section 3, the statistical behavior for a more general constraint is obtained. In Section 4, we speculate on the possible relationship with some economic systems [3-9] in which the Gamma distributions are also obtained. A formula for the volume of high-dimensional symmetrical bodies is proposed in Section 5. The last Section 6 contains our conclusions.

\section{Recalling Some Results}

\subsection{Linear constraint}

Let us assume $N$ agents interacting in an open economy, each one with coordinate $x_{i}, i=1, \ldots, N$, with $x_{i} \geq 0$ representing the wealth or money of the agent $i$, and a total available amount of money $E$. The additive linear constraint reads:

$$
x_{1}+x_{2}+\cdots+x_{N-1}+x_{N} \leq E
$$

The result here explained for an open economy is also obtained when constraint (1) is an equality, i.e., when the economy is closed [10]. Under random evolution rules for the exchanging of money among agents [3], let us suppose that this system evolves in the interior of the $N$-dimensional pyramid given by Equation (1). We can suppose that the state or the bank system of western societies plays in this model the role of a heat reservoir that supplies money instead of energy. The formula for the volume $V_{N}(E)$ of an equilateral $N$-dimensional pyramid formed by $N+1$ vertices linked by $N$ perpendicular sides of 
length $E$ is

$$
V_{N}(E)=\frac{E^{N}}{N !}
$$

If each point on the $N$-dimensional pyramid is equiprobable, then the probability $f\left(x_{i}\right) d x_{i}$ of finding the agent $i$ with money $x_{i}$, with normalization condition $\int_{0}^{E} f\left(x_{i}\right) d x_{i}=1$, is proportional to the volume formed by all the points into the $(N-1)$-dimensional pyramid having the $i$ th-coordinate equal to $x_{i}$. Then $f\left(x_{i}\right)$ verifies

$$
f\left(x_{i}\right)=\frac{V_{N-1}\left(E-x_{i}\right)}{V_{N}(E)}
$$

If we call $\epsilon$ the mean wealth per agent, $E=N \epsilon$, then in the limit of large $N(N \rightarrow \infty)$, we have

$$
\lim _{N \gg 1} \frac{V_{N-1}(E-x)}{V_{N}(E)}=\frac{1}{\epsilon} e^{-x / \epsilon}
$$

where the index $i$ has been removed because the distribution is the same for each agent, and thus the wealth distribution can be obtained by averaging over all the agents,

$$
f(x) d x=\epsilon^{-1} e^{-x / \epsilon} d x
$$

This Boltzmann-Gibbs distribution has been found to fit the real distribution of incomes in western societies [3].

\subsection{Quadratic constraint}

Now let us suppose a one-dimensional ideal gas of $N$ non-identical classical particles with masses $m_{i}$, with $i=1, \ldots, N$, and total maximum energy $E$. If particle $i$ has a momentum $m_{i} v_{i}$, we define a kinetic energy:

$$
K \equiv p_{i}^{2} \equiv \frac{1}{2} m_{i} v_{i}^{2}
$$

where $p_{i}$ is the square root of the kinetic energy. Then the quadratic constraint reads:

$$
p_{1}^{2}+p_{2}^{2}+\cdots+p_{N-1}^{2}+p_{N}^{2} \leq E
$$

The distribution for $p_{i}$ here derived for an open system is also obtained when constraint (7) is an equality, i.e., when the energy is fixed [11]. When a finite number of particles are present in the system the asymptotic distribution maximizes the Tsallis entropy [12]. In the case with constraint (7) the system has accessible states with different energy, which is supposed to be supplied by a heat reservoir. These states are all those enclosed into the volume of the $N$-sphere given by Equation (7), with radius $E^{1 / 2}$. The formula for the volume $V_{N}(R)$ of an $N$-sphere of radius $R$ is

$$
V_{N}(R)=\frac{\pi^{\frac{N}{2}}}{\Gamma\left(\frac{N}{2}+1\right)} R^{N}
$$

where $\Gamma(\cdot)$ is the Gamma function. If we suppose that each point into the $N$-sphere is equiprobable, then the probability $f\left(p_{i}\right) d p_{i}$ of finding the particle $i$, with coordinate $p_{i}$ (energy $p_{i}^{2}$ ) and normalization 
condition $\int_{-R}^{R} f\left(p_{i}\right) d p_{i}=1$, is proportional to the volume formed by all the points into the $(N-1)$-sphere having the $i$ th-coordinate equal to $p_{i}$. Then $f\left(p_{i}\right)$ verifies

$$
f\left(p_{i}\right)=\frac{V_{N-1}\left(\left(E-p_{i}^{2}\right)^{1 / 2}\right)}{V_{N}\left(E^{1 / 2}\right)}
$$

If we call $\epsilon$ the mean energy per particle, $E=N \epsilon$, then in the limit of large $N(N \rightarrow \infty)$, we have

$$
\lim _{N \gg 1} \frac{V_{N-1}\left(\left(E-p^{2}\right)^{1 / 2}\right)}{V_{N}\left(E^{1 / 2}\right)}=\sqrt{\frac{1}{2 \pi}} \epsilon^{-1 / 2} e^{-p^{2} / 2 \epsilon}
$$

where the index $i$ has been removed because the distribution is the same for each particle. Thus the asymptotic distribution

$$
f(p)=\sqrt{\frac{1}{2 \pi}} \epsilon^{-1 / 2} e^{-p^{2} / 2 \epsilon}
$$

can be obtained by averaging over all the particles. If the change of variables $p=\sqrt{\frac{m}{2}} v$ is performed, with $v$ the generic velocity of a particle, then the Maxwellian distribution is just derived from geometrical arguments.

\section{Multi-Agent Systems and Equiprobability: General Derivation of the Asymptotic Distribution}

In this section, we address the same problem above presented but in a general way. Let $b$ be a positive real constant (cases $b=1,2$ have been indicated in the former section). If we have a set of positive variables $\left(x_{1}, x_{2}, \ldots, x_{N}\right)$ verifying the constraint

$$
x_{1}^{b}+x_{2}^{b}+\cdots+x_{N-1}^{b}+x_{N}^{b} \leq E
$$

with an adequate mechanism assuring the equiprobability of all the possible states $\left(x_{1}, x_{2}, \ldots, x_{N}\right)$ into the volume given by expression (12), will we have for the generic variable $x$ the distribution

$$
f(x) d x \sim \epsilon^{-1 / b} e^{-x^{b} / b \epsilon} d x
$$

when we average over the ensemble in the limit $N, E \rightarrow \infty$, with $E=N \epsilon$, and constant $\epsilon$ ?. Now it is shown that the answer is affirmative. Similarly, we claim that if the weak inequality (12) is transformed in equality the result will be the same, as it has been proved for the cases $b=1,2$ in References [10, 11].

From the cases $b=1,2$, (see Equations (3) and (9)), we can extrapolate the general formula that will give us the statistical behavior $f(x)$ of the generic variable $x$, when the system runs equiprobably into the volume defined by a constraint of type (12). The probability $f(x) d x$ of finding an agent with generic coordinate $x$ is proportional to the volume $V_{N-1}\left(\left(E-x^{b}\right)^{1 / b}\right)$ formed by all the points into the $(N-1)$-dimensional symmetrical body limited by the constraint $\left(E-x^{b}\right)$. Thus, the $N$-dimensional volume can be written as

$$
V_{N}\left(E^{1 / b}\right)=\int_{0}^{E^{1 / b}} V_{N-1}\left(\left(E-x^{b}\right)^{1 / b}\right) d x
$$

Taking into account the normalization condition $\int_{0}^{E^{1 / b}} f(x) d x=1$, the expression for $f(x)$ is obtained:

$$
f(x)=\frac{V_{N-1}\left(\left(E-x^{b}\right)^{1 / b}\right)}{V_{N}\left(E^{1 / b}\right)}
$$


The $N$-dimensional volume, $V_{N}(b, \rho)$, of a $b$-symmetrical body with side of length $\rho$ is proportional to the term $\rho^{N}$ and to a coefficient $g_{b}(N)$ that depends on $N$ :

$$
V_{N}(b, \rho)=g_{b}(N) \rho^{N}
$$

The parameter $b$ indicates the original Equation (12) that defines the boundaries of the volume $V_{N}(b, \rho)$. Thus, for instance, from Equation (2), we have $g_{b=1}(N)=1 / N$ !.

Coming back to Equation (15), we can manipulate $V_{N}\left(\left(E-x^{b}\right)^{1 / b}\right)$ to obtain (the index $b$ is omitted in the formula of $V_{N}$ ):

$$
V_{N}\left(\left(E-x^{b}\right)^{1 / b}\right)=g_{b}(N)\left[\left(E-x^{b}\right)^{1 / b}\right]^{N}=g_{b}(N) E^{\frac{N}{b}}\left(1-\frac{x^{b}}{E}\right)^{\frac{N}{b}}
$$

If we suppose $E=N \epsilon$, then $\epsilon$ represents the mean value of $x^{b}$ in the collectivity, that is, $\epsilon=<x^{b}>$. If $N$ tends toward infinity, it results:

$$
\lim _{N \gg 1}\left(1-\frac{x^{b}}{E}\right)^{\frac{N}{b}}=e^{-x^{b} / b \epsilon}
$$

Thus,

$$
V_{N}\left(\left(E-x^{b}\right)^{1 / b}\right)=V_{N}\left(E^{1 / b}\right) e^{-x^{b} / b \epsilon}
$$

Substituting this last expression in formula (15), the exact form for $f(x)$ is found in the thermodynamic $\operatorname{limit}(N, E \rightarrow \infty)$ :

$$
f(x) d x=c_{b} \epsilon^{-1 / b} e^{-x^{b} / b \epsilon} d x
$$

with $c_{b}$ given by

$$
c_{b}=\frac{g_{b}(N-1)}{g_{b}(N) N^{1 / b}}
$$

Hence, the conjecture (13) is proved.

Doing a thermodinamical simile, we can calculate the dependence of $\epsilon$ on the temperature by differentiating the entropy with respect to the energy. The entropy can be written as $S=-k N \int_{0}^{\infty} f(x) \ln f(x) d x$, where $f(x)$ is given by Equation (20) and $k$ is the Boltzmann constant. If we recall that $\epsilon=E / N$, we obtain

$$
S(E)=\frac{k N}{b} \ln \left(\frac{E}{N}\right)+\frac{k N}{b}\left(1-b \ln c_{b}\right)
$$

where it has been used that $\epsilon=<x^{b}>=\int_{0}^{\infty} x^{b} f(x) d x$

The calculation of the temperature $T$ gives

$$
T^{-1}=\left(\frac{\partial S}{\partial E}\right)_{N}=\frac{k N}{b E}=\frac{k}{b \epsilon}
$$

Thus $\epsilon=k T / b$, a result that recovers the theorem of equipartition of energy for the quadratic case $b=2$. The distribution for all $b$ is finally obtained:

$$
f(x) d x=c_{b}\left(\frac{b}{k T}\right)^{1 / b} e^{-x^{b} / k T} d x
$$




\section{Gamma Distributions, Economic Gas Models and Geometry: A Speculation}

If we perform the change of variables $y=\epsilon^{-1 / b} x$ in the normalization condition of $f(x), \int_{0}^{\infty} f(x) d x=$ 1 , where $f(x)$ is expressed in (20), we find that

$$
c_{b}=\left[\int_{0}^{\infty} e^{-y^{b} / b} d y\right]^{-1}
$$

If we introduce the new variable $z=y^{b} / b$, the distribution $f(x)$ as function of $z$ reads:

$$
f(z) d z=\frac{c_{b}}{b^{1-\frac{1}{b}}} z^{\frac{1}{b}-1} e^{-z} d z
$$

Let us observe that the Gamma function appears in the normalization condition,

$$
\int_{0}^{\infty} f(z) d z=\frac{c_{b}}{b^{1-\frac{1}{b}}} \int_{0}^{\infty} z^{\frac{1}{b}-1} e^{-z} d z=\frac{c_{b}}{b^{1-\frac{1}{b}}} \Gamma\left(\frac{1}{b}\right)=1
$$

This implies that

$$
c_{b}=\frac{b^{1-\frac{1}{b}}}{\Gamma\left(\frac{1}{b}\right)}
$$

By using Mathematica the positive constant $c_{b}$ is plotted versus $b$ in Figure 1 . We see that $\lim _{b \rightarrow 0} c_{b}=\infty$, and that $\lim _{b \rightarrow \infty} c_{b}=1$. The minimum of $c_{b}$ is reached for $b=3.1605$, taking the value $c_{b}=0.7762$. Still further, we can calculate from Equation (28) the asymptotic dependence of $c_{b}$ on b:

$$
\begin{aligned}
\lim _{b \rightarrow 0} c_{b} & =\sqrt{\frac{1}{2 \pi}} \sqrt{b} e^{1 / b}\left(1-\frac{b}{12}+\cdots\right) \\
\lim _{b \rightarrow \infty} c_{b} & =b^{-1 / b}\left(1+\frac{\gamma}{b}+\cdots\right)
\end{aligned}
$$

where $\gamma$ is the Euler constant, $\gamma=0.5772$. The asymptotic function (29) is obtained after substituting in (28) the value of $\Gamma(1 / b)$ by $(1 / b-1)$ !, and performing the Stirling approximation on this last expression, knowing that $1 / b \rightarrow \infty$. The function (30) is found after looking for the first Taylor expansion terms of the Gamma function around the origin $x=0$. They can be derived from the Euler's reflection formula, $\Gamma(x) \Gamma(1-x)=\pi / \sin (\pi x)$. We obtain $\Gamma(x \rightarrow 0)=x^{-1}+\Gamma^{\prime}(1)+\cdots$. From here, recalling that $\Gamma^{\prime}(1)=-\gamma$, we get $\Gamma(1 / b)=b-\gamma+\cdots$, when $b \rightarrow \infty$. Although this last term of the Taylor expansion, $-\gamma$, is negligible we maintain it in expression (30). The only minimum of $c_{b}$ is reached for the solution $b=3.1605$ of the equation $\psi(1 / b)+\log b+b-1=0$, where $\psi(\cdot)$ is the digamma function (see Figure 1).

Let us now recall two interesting statistical economic models that display a statistical behavior given by distributions of the form (26), that is, the standard Gamma distributions with shape parameter $1 / b$,

$$
f(z) d z=\frac{1}{\Gamma\left(\frac{1}{b}\right)} z^{\frac{1}{b}-1} e^{-z} d z
$$


Figure 1. Normalization constant $c_{b}$ versus $b$, calculated from Equation (28). The asymptotic behavior is: $\lim _{b \rightarrow 0} c_{b}=\infty$, and $\lim _{b \rightarrow \infty} c_{b}=1$. This last asymptote is represented by the dotted line. The minimum of $c_{b}$ is reached for $b=3.1605$, taking the value $c_{b}=0.7762$.

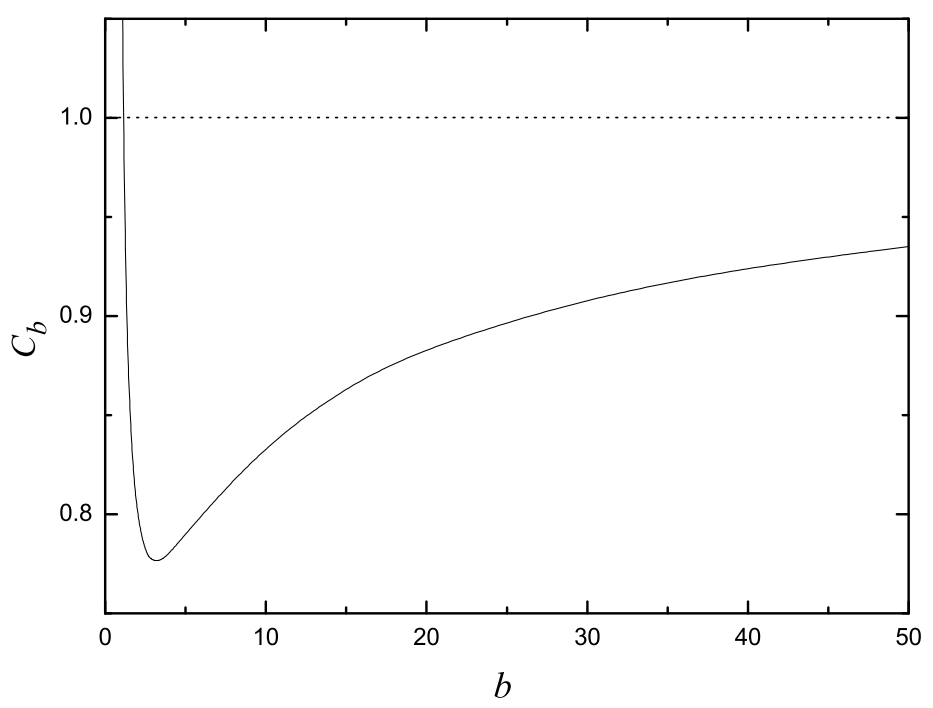

ECONOMIC MODEL A: The first one is the saving propensity model introduced by Chakraborti and Chakrabarti [4]. In this model a set of $N$ economic agents, having each agent $i$ (with $i=1,2, \cdots, N$ ) an amount of money, $u_{i}$, exchanges it under random binary $(i, j)$ interactions, $\left(u_{i}, u_{j}\right) \rightarrow\left(u_{i}^{\prime}, u_{j}^{\prime}\right)$, by the following the exchange rule:

$$
\begin{aligned}
& u_{i}^{\prime}=\lambda u_{i}+\epsilon(1-\lambda)\left(u_{i}+u_{j}\right) \\
& u_{j}^{\prime}=\lambda u_{j}+\bar{\epsilon}(1-\lambda)\left(u_{i}+u_{j}\right)
\end{aligned}
$$

with $\bar{\epsilon}=(1-\epsilon)$, and $\epsilon$ a random number in the interval $(0,1)$. The parameter $\lambda$, with $0<\lambda<1$, is fixed, and represents the fraction of money saved before carrying out the transaction. Let us observe that money is conserved, i.e., $u_{i}+u_{j}=u_{i}^{\prime}+u_{j}^{\prime}$, hence in this model the economy is closed. Defining the parameter $n(\lambda)$ as

$$
n(\lambda)=\frac{1+2 \lambda}{1-\lambda}
$$

and scaling the wealth of the agents as $\bar{z}=n u /\langle u\rangle$, with $\langle u\rangle$ representing the average money over the ensemble of agents, it is found that the asymptotic wealth distribution in this system obeys the standard Gamma distribution[5]

$$
f(\bar{z}) d \bar{z}=\frac{1}{\Gamma(n)} \bar{z}^{n-1} e^{-\bar{z}} d \bar{z}
$$

The case $n=1$, which means a null saving propensity, $\lambda=0$, recovers the model of Dragulescu and Yakovenko [3] in which the Gibbs distribution is observed. If we compare Equations (35) and (31), a close relationship between this economic model and the geometrical problem solved in the former 
section can be established. It is enough to make

$$
\begin{aligned}
& n=1 / b \\
& \bar{z}=z
\end{aligned}
$$

to have two equivalent systems. This means that, from Equation (36), we can calculate $b$ from the saving parameter $\lambda$ with the formula

$$
b=\frac{1-\lambda}{1+2 \lambda}
$$

As $\lambda$ takes its values in the interval $(0,1)$, then the parameter $b$ also runs in the same interval $(0,1)$. On the other hand, recalling that $z=x^{b} / b \epsilon$, we can get the equivalent variable $x$ from Equation (37),

$$
x=\left[\frac{\epsilon}{<u>} u\right]^{1 / b}
$$

where $\epsilon$ is a free parameter that determines the mean value of $x^{b}$ in the equivalent geometrical system. Formula (39) means to perform the change of variables $u_{i} \rightarrow x_{i}$, with $i=1,2, \cdots, N$, for all the particles/agents of the ensemble. Then, we conjecture that the economic system represented by the generic pair $(\lambda, u)$, when it is transformed in the geometrical system given by the generic pair $(b, x)$, as indicated by the rules (38) and (39), runs in an equiprobable form on the surface defined by the relationship (12), where the inequality has been transformed in equality. This last detail is due to the fact the economic system is closed, and then it conserves the total money, whose equivalent quantity in the geometrical problem is $E$. If the economic system were open, with an upper limit in the wealth, then the transformed system would evolve in an equiprobable way over the volume defined by the inequality (12), although its statistical behavior would continue to be the same as it has been proved for the cases $b=1,2$ in $[10,11]$.

ECONOMIC MODEL B: The second one is a model introduced in [6]. In this model a set of $N$ economic agents, having each agent $i$ (with $i=1,2, \cdots, N$ ) an amount of money, $u_{i}$, exchanges it under random binary $(i, j)$ interactions, $\left(u_{i}, u_{j}\right) \rightarrow\left(u_{i}^{\prime}, u_{j}^{\prime}\right)$, by the following the exchange rule:

$$
\begin{aligned}
& u_{i}^{\prime}=u_{i}-\Delta u \\
& u_{j}^{\prime}=u_{j}+\Delta u
\end{aligned}
$$

where

$$
\Delta u=\eta\left(x_{i}-x_{j}\right) \epsilon \omega x_{i}-\left[1-\eta\left(x_{i}-x_{j}\right)\right] \epsilon \omega x_{j}
$$

with $\epsilon$ a continuous uniform random number in the interval $(0,1)$. When this variable is transformed in a Bernouilli variable, i.e., a discrete uniform random variable taking on the values 0 or 1 , we have the model studied by Angle [7], that gives very different asymptotic results. The exchange parameter, $\omega$, represents the maximum fraction of wealth lost by one of the two interacting agents $(0<\omega<1)$. Whether the agent who is going to loose part of the money is the $i$-th or the $j$-th agent, depends nonlinearly on $\left(x_{i}-x_{j}\right)$, and this is decided by the random dichotomous function $\eta(t): \eta(t>0)=1$ (with additional probability $1 / 2$ ) and $\eta(t<0)=0$ (with additional probability $1 / 2$ ). Hence, when $x_{i}>x_{j}$, the value $\eta=1$ produces a wealth transfer from agent $i$ to agent $j$ with 
probability $1 / 2$, and when $x_{i}<x_{j}$, the value $\eta=0$ produces a wealth transfer from agent $j$ to agent $i$ with probability $1 / 2$. Defining in this case the parameter $n(\omega)$ as

$$
n(\omega)=\frac{3-2 \omega}{2 \omega}
$$

and scaling the wealth of the agents as $\bar{z}=n u /\langle u\rangle$, with $\langle u\rangle$ representing the average money over the ensemble of agents, it is found that the asymptotic wealth distribution in this system obeys the standard Gamma distribution[6]

$$
f(\bar{z}) d \bar{z}=\frac{1}{\Gamma(n)} \bar{z}^{n-1} e^{-\bar{z}} d \bar{z}
$$

The case $n=1$, which means an exchange parameter $\omega=3 / 4$, recovers the model of Dragulescu and Yakovenko [3] in which the Gibbs distribution is observed. If we compare Equations (44) and (31), a close relationship between this economic model and the geometrical problem solved in the last section can be established. It is enough to make

$$
\begin{aligned}
& n=1 / b \\
& \bar{z}=z
\end{aligned}
$$

to have two equivalent systems. This means that, from Equation (45), we can calculate $b$ from the exchange parameter $\omega$ with the formula

$$
b=\frac{2 \omega}{3-2 \omega}
$$

As $\omega$ takes its values in the interval $(0,1)$, then the parameter $b$ runs in the interval $(0,2)$. It is curious to observe that in this model the interval $\omega \in(3 / 4,1)$ maps on $b \in(1,2)$, a fact that does not occur in MODEL A. On the other hand, recalling that $z=x^{b} / b \epsilon$, we can get the equivalent variable $x$ from Equation (46),

$$
x=\left[\frac{\epsilon}{<u>} u\right]^{1 / b}
$$

where $\epsilon$ is a free parameter that determines the mean value of $x^{b}$ in the equivalent geometrical system. Formula (48) means to perform the change of variables $u_{i} \rightarrow x_{i}$, with $i=1,2, \cdots, N$, for all the particles/agents of the ensemble. Then, we conjecture that the economic system represented by the generic pair $(\lambda, u)$, when it is transformed in the geometrical system given by the generic pair $(b, x)$, as indicated by the rules (47) and (48), runs in an equiprobable form on the surface defined by the relationship (12), where the inequality has been transformed in equality. As explained above, this last detail is due to the fact the economic system is closed, and then it conserves the total money, whose equivalent quantity in the geometrical problem is $E$. If the economic system were open, with an upper limit in the wealth, then the transformed system would evolve in an equiprobable way over the volume defined by the inequality (12), although its statistical behavior would continue to be the same as it has been proved for the cases $b=1,2$ in References [10, 11].

\section{Other Geometrical Questions}

We shall proceed now to derive an asymptotic formula $(N \rightarrow \infty)$ for the volume of the $N$-dimensional symmetrical body enclosed by the surface

$$
x_{1}^{b}+x_{2}^{b}+\cdots+x_{N-1}^{b}+x_{N}^{b}=E
$$


The linear dimension $\rho$ of this volume, i.e., the length of one of its sides verifies $\rho \sim E^{1 / b}$. As argued in Equation (16), the $N$-dimensional volume, $V_{N}(b, \rho)$, is proportional to the term $\rho^{N}$ and to a coefficient $g_{b}(N)$ that depends on $N$. Thus,

$$
V_{N}(b, \rho)=g_{b}(N) \rho^{N}
$$

where the characteristic $b$ indicates the particular boundary given by Equation (49).

For instance, from Equation (2), we can write in a formal way:

$$
g_{b=1}(N)=\frac{1^{\frac{N}{1}}}{\Gamma\left(\frac{N}{1}+1\right)}
$$

From Equation (8), if we take the diameter, $\rho=2 R$, as the linear dimension of the $N$-sphere, we obtain:

$$
g_{b=2}(N)=\frac{\left(\frac{\pi}{4}\right)^{\frac{N}{2}}}{\Gamma\left(\frac{N}{2}+1\right)}
$$

These expressions (51) and (52) suggest a possible general formula for the factor $g_{b}(N)$, let us say

$$
g_{b}(N)=\frac{a^{\frac{N}{b}}}{\Gamma\left(\frac{N}{b}+1\right)}
$$

where $a$ is a $b$-dependent constant to be determined. For example, $a=1$ for $b=1$ and $a=\pi / 4$ for $b=2$.

In order to find the dependence of $a$ on the parameter $b$, the regime $N \rightarrow \infty$ is supposed. Applying Stirling approximation for the factorial $\left(\frac{N}{b}\right)$ ! in the denominator of expression (53), and inserting it in expression (21), it is straightforward to find out the relationship:

$$
c_{b}=(a b)^{-1 / b}
$$

From here and formula (28), we get:

$$
a=\left[\Gamma\left(\frac{1}{b}+1\right)\right]^{b}
$$

that recovers the exact results for $b=1,2$. The behavior of $a$ is monotonous decreasing when $b$ is varied from $b=0$, where $a$ diverges as $a \sim 1 / b+\cdots$, up to the limit $b \rightarrow \infty$, where $a$ decays asymptotically toward the value $a_{\infty}=e^{-\gamma}=0.5614$.

Hence, the formula for $g_{b}(N)$ is obtained:

$$
g_{b}(N)=\frac{\Gamma\left(\frac{1}{b}+1\right)^{N}}{\Gamma\left(\frac{N}{b}+1\right)}
$$

It would be also possible to multiply this last expression (56) by a general polynomial $K(N)$ in the variable $N$, and all the derivation done from Equation (53) would continue to be correct. We omit this possibility in our calculations. For a fixed $N$, we have that $g_{b}(N)$ increases monotonously from $g_{b}(N)=0$, for $b=0$, up to $g_{b}(N)=1$, in the limit $b \rightarrow \infty$ (see Figure 2). For a fixed $b$, we have that $g_{b}(N)$ decreases monotonously from $g_{b}(N)=1$, for $N=1$, up to $g_{b}(N)=0$, in the limit $N \rightarrow \infty$ (see Figure 3). 
Figure 2. The factor $g_{b}(N)$ versus $b$ for $N=10,40,100$, calculated from Equation (56). Observe that $g_{b}(N)=0$ for $b=0$, and $\lim _{b \rightarrow \infty} g_{b}(N)=1$.

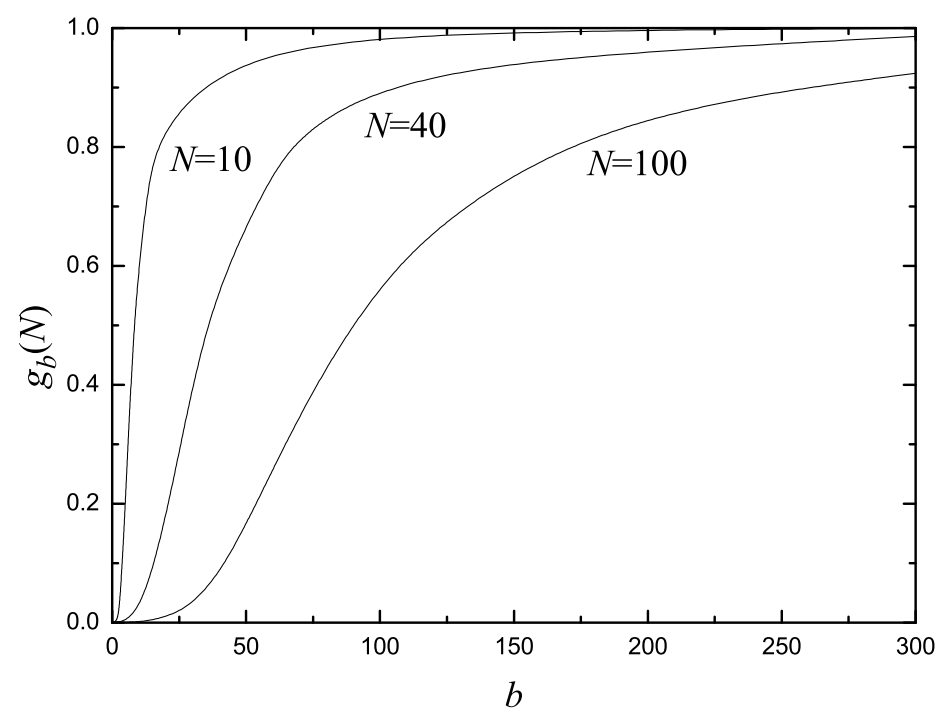

Figure 3. The factor $g_{b}(N)$ versus $N$ for $b=10,40,100$, calculated from Equation (56). Observe that $g_{b}(N)=1$ for $N=1$, and $\lim _{N \rightarrow \infty} g_{b}(N)=0$.

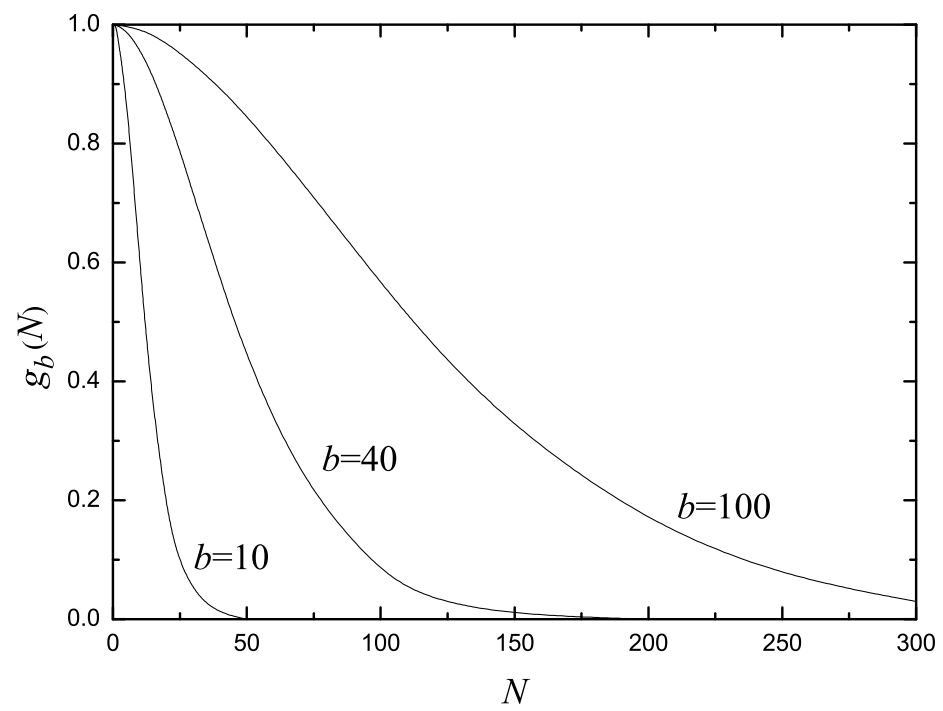

The final result for the volume of an $N$-dimensional symmetrical body of characteristic $b$ given by the boundary (49) reads:

$$
V_{N}(b, \rho)=\frac{\Gamma\left(\frac{1}{b}+1\right)^{N}}{\Gamma\left(\frac{N}{b}+1\right)} \rho^{N}
$$


with $\rho \sim E^{1 / b}$.

\section{Conclusions}

In this work, we have considered a general multi-agent open system verifying an additive constraint. Its statistical behavior has been derived from geometrical arguments. The Maxwellian and the Boltzmann-Gibbs distributions are particular cases of this type of systems. Also, other multi-agent economy models, such as the Dragalescu and Yakovenko's model [3], the Chakraborti and Chakrabarti's model [4] and the modified Angle's model [6], show similar statistical behaviors compared to our general system. This fact fosters a geometrical interpretation of all those models. This geometrical speculation allows us to suggest the equivalence with the Chakraborti and Chakrabarti's model when the geometrical characteristic $b$ of our model runs in the interval $(0,1)$. The equivalence with the modified Angle's model is suggested when $b$ varies in the interval $(0,2)$. As a particular case of both types of models, the Dragulescu and Yakovenko's model is obtained for $b=1$.

We have not found in the literature other multi-agent models to establish an equivalence with our system in the range $b \in(2, \infty)$. This point remains an open question and a challenge that will probably trigger other works in this direction.

\section{Acknowledgements}

The authors acknowledge some financial support from Spanish DGICYT Projects FIS2006-12781-C02-01.

\section{References}

1. Mantegna, R.; Stanley, H.E. An Introduction to Econophysics: Correlations and Complexity in Finance; Cambridge University Press: Cambridge, UK, 1999.

2. Yakovenko, V.M. Econophysics, Statistical Mechanics Approach to. In Encyclopedia of Complexity and System Science; Meyers, R.A., Ed.; Springer: Berlin, Germany, 2009; pp. 2800-2826.

3. Dragulescu, A.; Yakovenko, V.M. Statistical mechanics of money. Eur. Phys. J. 2000, B17, 723-729.

4. Chakraborti, A.; Chakrabarti, B.K. Statistical mechanics of money: How saving propensity affects its distribution. Eur. Phys. J. B 2000, 17, 167-170.

5. Patriarca, M.; Chakraborti, A.; Kaski, K. Statistical model with a standard Gamma distribution. Phys. Rev. E 2004, 70, 016104-016105.

6. Patriarca, M.; Heinsalu, E.; Chakraborti, A. The ABCD's of statistical many-agent economy models. 2006, arXiv:physics/0611245.

7. Angle, J. The inequality process as a wealth maximizing process. Physica A 2006, 367, 388-414, (references therein).

8. Gonzalez-Estevez, J.; Cosenza, M.G.; Lopez-Ruiz, R.; Sanchez, J.R. Pareto and Boltzmann-Gibbs behaviors in a deterministic multi-agent system. Physica A 2008, 387, 4637-4642.

9. Pellicer-Lostao, C.; Lopez-Ruiz, R. Economic models with chaotic money exchange. In Proceedings of the ICCS 2009, Baton Rouge, LA, USA, 2009; Part I, pp. 43-52. 
10. Lopez-Ruiz, R.; Sañudo, J.; Calbet, X. Geometrical derivation of the Boltzmann factor. Am. J. Phys. 2008, 76, 780-781.

11. Lopez-Ruiz, R.; Calbet, X. Derivation of the Maxwellian distribution from the microcanonical ensemble. Am. J. Phys. 2007, 75, 752-753.

12. Harremoës, P. Projections maximizing Tsallis entropy. AIP Conf. Proc. 2007, 965, 90-95.

(c) 2009 by the authors; licensee Molecular Diversity Preservation International, Basel, Switzerland. This article is an open-access article distributed under the terms and conditions of the Creative Commons Attribution license http://creativecommons.org/licenses/by/3.0/. 